\title{
Maximal exercise capacity in patients with obstructive sleep apnoea syndrome: a systematic review and meta-analysis
}

\author{
Monique Mendelson ${ }^{1,2,3,4}$, Mathieu Marillier ${ }^{1,2,3,4}$, Sébastien Bailly, 2,3, \\ Patrice Flore ${ }^{1,2,3}$, Jean-Christian Borel ${ }^{1,2,3}$, Isabelle Vivodtzev (1) 1,2,3 \\ Stéphane Doutreleau ${ }^{1,2,3}$, Renaud Tamisier (1) ${ }^{1,2,3}$, Jean-Louis Pépin ${ }^{1,2,3,5}$ and \\ Samuel Verges ${ }^{1,2,3,5}$
}

Affiliations: ${ }^{1} \mathrm{HP} 2$ Laboratory, University Grenoble Alpes, Grenoble, France. ${ }^{2}$ Inserm U1042, Grenoble, France. ${ }^{3}$ Grenoble Alps University Hospital, Grenoble, France. ${ }^{4}$ Both authors contributed equally and share the first authorship. ${ }^{5}$ Both authors share senior authorship.

Correspondence: Monique Mendelson, Laboratoire HP2, UM Sports Pathologies, Hôpital Sud, Avenue Kimberley, 38434 Echirolles, France. E-mail: mmendelsonachu-grenoble.fr

@ERSpublications

Maximal exercise capacity as a reflection of total body health is reduced in patients with obstructive sleep apnoea http://ow.ly/EdaD30jAE7k

Cite this article as: Mendelson M, Marillier M, Bailly S, et al. Maximal exercise capacity in patients with obstructive sleep apnoea syndrome: a systematic review and meta-analysis. Eur Respir J 2018; 51: 1702697 [https://doi.org/10.1183/13993003.02697-2017].

ABSTRACT Maximal aerobic capacity is a strong health predictor and peak oxygen consumption ( $V^{\prime} \mathrm{O}_{2}$ peak) is considered a reflection of total body health. No systematic reviews or meta-analyses to date have synthesised the existing data regarding $V^{\prime} \mathrm{O}_{2}$ peak in patients with obstructive sleep apnoea (OSA).

A systematic review of English and French articles using PubMed/MEDLINE and Embase included studies assessing $V^{\prime} \mathrm{O}_{2}$ peak in OSA patients either in $\mathrm{mL} \cdot \mathrm{kg}^{-1} \cdot \mathrm{min}^{-1}$ compared with controls or in $\%$ predicted. Two independent reviewers analysed the studies, extracted the data and assessed the quality of evidence.

Mean $V^{\prime} \mathrm{O}_{2}$ peak expressed in $\mathrm{mL} \cdot \mathrm{kg}^{-1} \cdot \mathrm{min}^{-1}$ was significantly lower in patients with OSA than in controls (mean difference $-2.7 \mathrm{~mL} \cdot \mathrm{kg}^{-1} \cdot \mathrm{min}^{-1} ; \mathrm{p}<0.001 ; \mathrm{n}=850$ ). This reduction in $V^{\prime} \mathrm{O}_{2}$ peak was found to be larger in non-obese patients (body mass index $<30 \mathrm{~kg} \cdot \mathrm{m}^{-2}$ ). Mean $V^{\prime} \mathrm{O}_{2}$ peak $\%$ pred was $89.9 \%$ in OSA patients $(n=643)$.

OSA patients have reduced maximal aerobic capacity, which can be associated with increased cardiovascular risks and reduced survival in certain patient subgroups. Maximal exercise testing can be useful to characterise functional limitation and to evaluate health status in OSA patients.

This article has supplementary material available from erj.ersjournals.com

Received: Dec 252017 | Accepted after revision: April 132018

This study was registered on the Prospero registry (CRD42017057319)

Copyright OERS 2018 


\section{Introduction}

Obstructive sleep apnoea (OSA) is a common clinical condition characterised by repeated episodes of apnoea and hypopnoea during sleep. Sleep fragmentation and chronic intermittent hypoxia induce intermediate mechanisms, such as activation of the sympathetic nervous system [1], oxidative stress and systemic inflammation [2], which are responsible for cardiometabolic morbidity [3] and mortality.

Maximal exercise capacity testing using an incremental whole-body (e.g. cycling) protocol with complementary respiratory gas-exchange measurements is considered the gold standard when assessing aerobic capacity [4]. Maximal exercise capacity is directly related to the integrated function of numerous systems and is therefore considered a reflection of total body health. The main measurement or surrogate marker of risk of these tests is peak oxygen uptake ( $V^{\prime} \mathrm{O}_{2}$ peak). In addition, maximal cardiorespiratory responses at peak exercise can provide useful information in interpreting exercise limitations.

In a growing number of studies, reduced maximal exercise capacity is associated with an increased risk of cardiovascular disease and all-cause mortality. This observation has been made in healthy men and women; those with suspected or known cardiovascular disease; and those with comorbid conditions including obesity, hypertension and lipid abnormalities [5-8]. Furthermore, recent studies have expressed maximal exercise capacity in the context of survival benefit per metabolic equivalence (MET), which is a multiple of the resting metabolic rate approximating $3.5 \mathrm{~mL} \cdot \mathrm{kg}^{-1} \cdot \mathrm{min}^{-1}$. These studies have shown that each 1-MET higher maximal exercise capacity is associated with considerable (10-25\%) improvement in survival [9].

The evaluation of maximal exercise capacity in OSA patients can be relevant when assessing functional limitations due to the disease as well as the associated systemic consequences (e.g. cardiovascular and metabolic alterations). Furthermore, this type of evaluation can be used as an objective outcome to assess treatment efficiency and for patient follow-up. Nevertheless, the impact of OSA on maximal exercise capacity is still debated. Previous studies have yielded conflicting results, with some studies showing no impairment in maximal exercise capacity in OSA patients [10-12] and others showing reduced maximal exercise capacity [13-16] compared to control subjects. These studies have several methodological limitations that make generalisation of findings difficult owing to the presence of co-morbidities, varying levels of OSA severity and low sample sizes.

To date, no meta-analysis has been conducted to summarise findings regarding the maximal exercise capacity of OSA patients compared with controls. Therefore, the main objective of this systematic review and meta-analysis was to determine if maximal aerobic capacity is reduced in OSA patients. A secondary aim was to investigate maximal exercise capacity as a function of OSA severity and age and to explore the cardiorespiratory responses to maximal exercise in patients with OSA.

\section{Methods}

The Preferred Reporting Items for Systematic Reviews and Meta-analyses (PRISMA) statement and recommendations were followed for this meta-analysis (www.prisma-statement.org) (the PRISMA checklist can be found in supplementary table S1) [17] and the trial was registered on the Prospero registry (CRD42017057319).

\section{Search strategy and data sources}

A systematic literature review was conducted to identify manuscripts which investigated maximal exercise capacity in OSA patients. The web-based literature search included PubMed/MEDLINE and Embase databases. Search terms were selected to reflect the condition and outcome parameters. Search terms included a combination of text word terms and medical subject headings (MeSH) or Emtree terms (the sample search strategy is outlined in the supplementary material). For the condition, search terms included "sleep apnoea syndromes" [MeSH] OR "sleep apnoea, obstructive" [MeSH] OR "sleep disordered breathing", "exercise tolerance" [MeSH] OR “exercise test” [MeSH] OR "cardiorespiratory fitness" [MeSH] OR "oxygen consumption" [MeSH] OR physical fitness OR aerobic capacity. Terms were searched in all possible combinations using Boolean logical operators (AND, OR, NOT). Additionally, a manual search of bibliographies of included articles was conducted to identify relevant references which may not have been found by the automated search. Obtained references were indexed and managed using EndNote X7 (Thomson Reuters, New York, NY, USA).

\section{Eligibility criteria}

The following criteria were required for selection: 1) original research investigations; 2) conducted in humans; 3) conducted in adults; 4) including patients diagnosed with OSA of at least mild severity (apnoea-hypopnoea index $(\mathrm{AHI}) \geqslant 5$ events $\cdot \mathrm{h}^{-1}$ ) based on polysomnography or polygraphy; 5) including untreated OSA patients; 6) assessing aerobic capacity (maximal oxygen consumption $\left(V^{\prime} \mathrm{O}_{2} \max \right)$ or $V^{\prime} \mathrm{O}_{2}$ peak) by means of a graded exercise test to volitional exhaustion; 7) reporting measures as $V^{\prime} \mathrm{O}_{2}$ peak in 
$\%$ predicted or presenting results in $\mathrm{mL} \cdot \mathrm{kg}^{-1} \cdot \mathrm{min}^{-1}$ when comparing patients with OSA and controls; and 8) published in a peer-reviewed journal up to February 2017. We excluded studies that included patients with heart failure because of the known effect of heart failure on maximal exercise capacity [18]. Only articles in English and French were retained.

\section{Data items}

Reviewing procedure and data extraction

Database searches were first conducted in February 2017. Titles and/or abstracts of studies retrieved using the search strategy and from additional sources were screened independently by two review authors (MMe and $\mathrm{MMa}$ ) to identify studies that potentially met the inclusion criteria outlined above. Irrelevant references were removed. Potentially relevant studies were further assessed by obtaining and reading the full text and re-checking the pre-specified eligibility criteria. The full text of these potentially eligible studies was retrieved independently and assessed for eligibility by two review team members. Any disagreements over the eligibility of particular studies were resolved through discussion with a third reviewer (SV). Data extraction was then performed for retained studies.

For each reference, the following variables were systematically extracted and entered into a summary table: 1) author, year; 2) participants; 3) AHI cut-off; 4) sample size; 5) age; 6) body mass index (BMI); 7) study design; 8) outcomes; and 9) main findings. A summary of the studies screened, assessed for eligibility and included is presented in figure 1 (for $V^{\prime} \mathrm{O}_{2}$ peak compared with controls in $\mathrm{mL} \cdot \mathrm{kg}^{-1} \cdot \mathrm{min}^{-1}$ ) and figure 2 (for $V^{\prime} \mathrm{O}_{2}$ peak \% pred in OSA patients only).

\section{Methodological quality assessment}

The quality of the studies was peer-reviewed by MMe and MMa using a modified version of the Newcastle-Ottawa Scale (NOS) for observational studies (supplementary material) [19]. Disagreements were resolved by consensus. For the NOS, a system of points (stars) was given to the eligible categories of sample selection criteria, comparability on the basis of the design or analysis, and evaluation of outcome. The scores varied depending on the study design and ranged from 0 to 8 . Studies with scores above the median were classified as high-quality studies [20].

\section{Statistical analysis}

All included studies in the primary selection were included in the meta-analysis. For the main objective, two approaches were considered according to the units of $V^{\prime} \mathrm{O}_{2}$ peak. If $V^{\prime} \mathrm{O}_{2}$ peak was expressed as

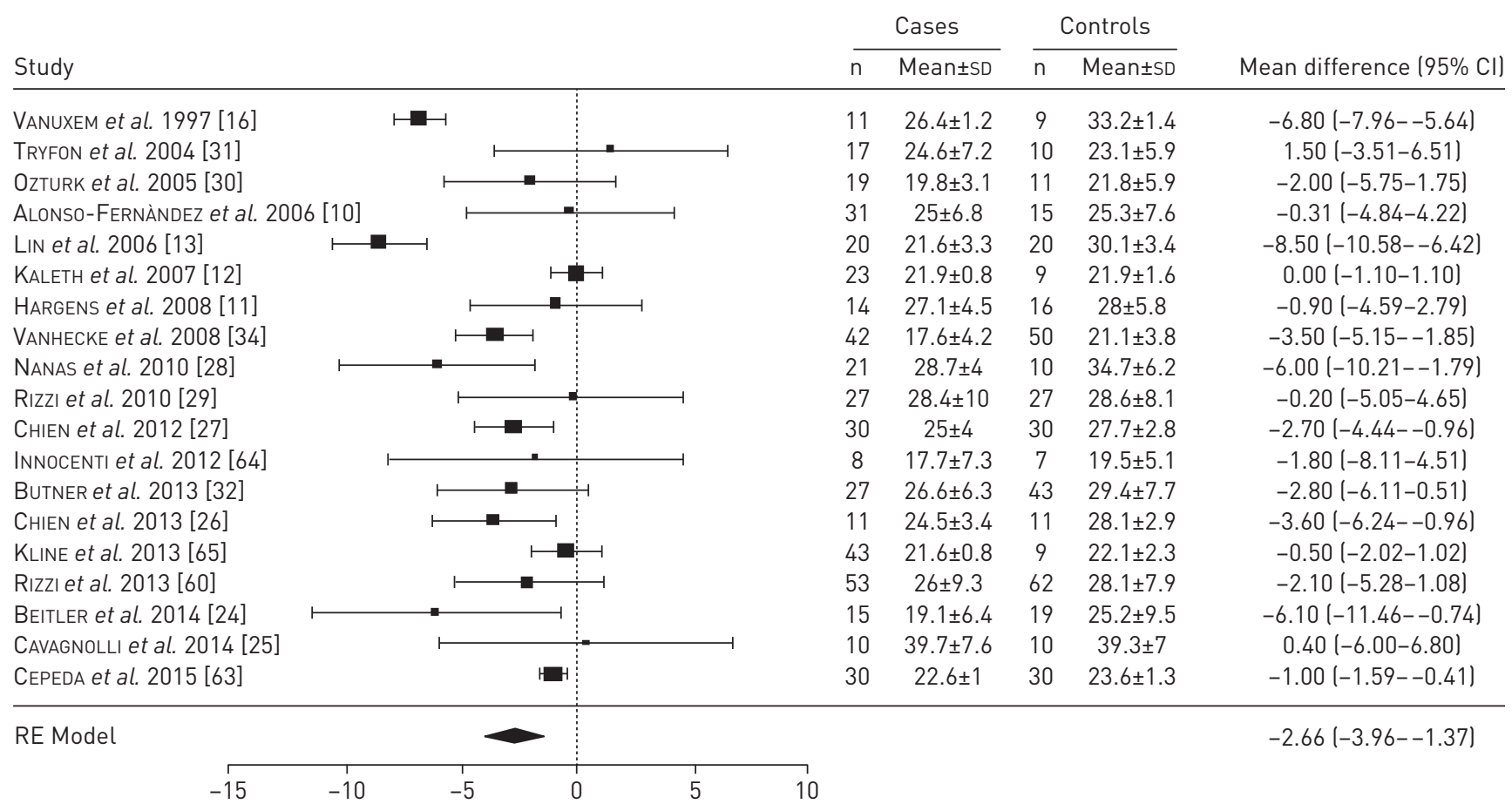

FIGURE 1 Forest plot for mean difference in peak oxygen consumption $\left(V^{\prime} \mathrm{O}_{2}\right.$ peak $)$ in $\mathrm{mL} \cdot \mathrm{kg}^{-1} \cdot \mathrm{min}^{-1}$ between patients with obstructive sleep apnoea and controls. The diamond reflects the $95 \%$ confidence interval of the pooled estimate of mean difference. RE: random effects. 


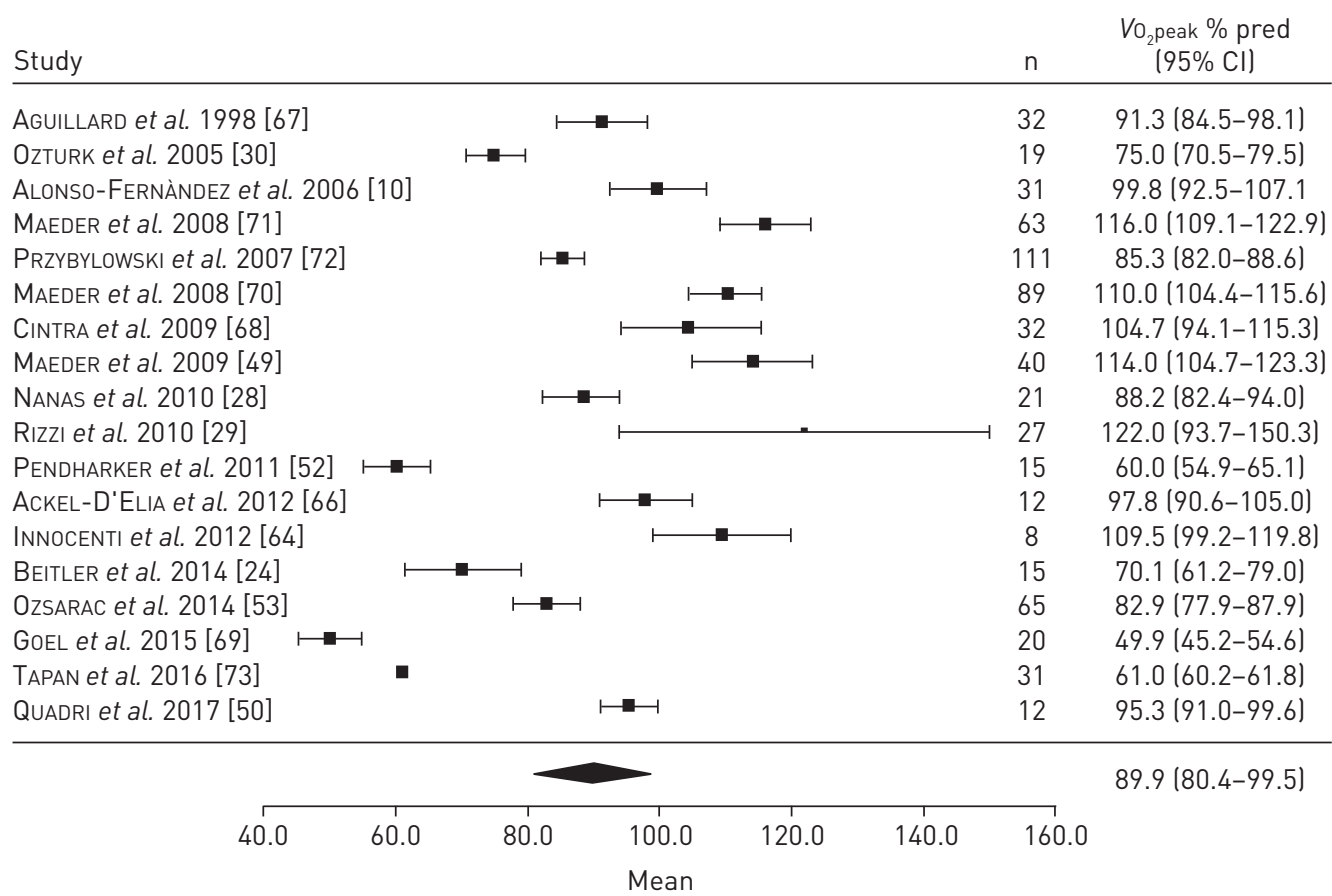

FIGURE 2 Forest plot for mean peak oxygen consumption $\left(V^{\prime}{ }{ }_{2}\right.$ peak $)$ in $\%$ predicted in patients with obstructive sleep apnoea.

$\mathrm{mL} \cdot \mathrm{kg}^{-1} \cdot \mathrm{min}^{-1}$, the mean difference was used to compare maximal exercise capacity between OSA patients and controls. For $V^{\prime} \mathrm{O}_{2}$ peak in $\%$ pred, we reported the mean value in OSA patients. A DerSimonian and Laird random effects meta-analysis model was used for $V^{\prime} \mathrm{O}_{2}$ peak in $\mathrm{mL} \cdot \mathrm{kg}^{-1} \cdot \mathrm{min}^{-1}$ to combine weighted mean differences [21]. The heterogeneity between studies was measured using the $\mathrm{I}^{2}$ inconsistency index, which provides an estimation of the variability due to heterogeneity rather than chance. An $\mathrm{I}^{2}$ index $>60 \%$ reflects increasing heterogeneity [22]. To investigate the heterogeneity, sensitivity analyses were performed using subgroups classified according to BMI, AHI and age. Finally, the robustness of the results was assessed using sensitivity analyses by leaving out one study at a time, and the absence of selection bias was assessed using funnel plots. The presence or the absence of asymmetry in the funnel plot was assessed using the Egger test. There was no exclusion of studies based on methodological quality assessment results. Meta-analyses were carried out using the $\mathrm{R}$ package metafor in the RStudio software (RStudio v 1.0.136) [23]. A p-value threshold of 0.05 was considered for statistical significance.

\section{Results}

The study selection process is presented in figure 3. The search of MEDLINE and Embase databases provided 1159 citations. After adjusting for duplicates, 1026 remained. Of these, 936 studies were discarded because a review of the abstracts indicated that these papers clearly did not meet the criteria. The full texts of the remaining 90 citations were examined in more detail and 61 did not meet the inclusion criteria. Of the 29 studies that met the inclusion criteria, 19 studies reported $V^{\prime} \mathrm{O}_{2}$ peak in $\mathrm{mL} \cdot \mathrm{kg}^{-1} \cdot \mathrm{min}^{-1}$ in OSA patients compared with controls and 18 reported $V^{\prime} \mathrm{O}_{2}$ peak in $\%$ pred values in OSA patients only.

\section{Main findings \\ $\mathrm{V}^{\prime} \mathrm{O}_{2}$ peak in $\mathrm{mL} \cdot \mathrm{kg}^{-1} \cdot \mathrm{min}^{-1}$ in patients with OSA compared with controls}

Mean $V^{\prime} \mathrm{O}_{2}$ peak expressed in $\mathrm{mL} \cdot \mathrm{kg}^{-1} \cdot \mathrm{min}^{-1}$ was significantly lower in patients with OSA than in controls (mean difference $-2.7 \mathrm{~mL} \cdot \mathrm{kg}^{-1} \cdot \mathrm{min}^{-1}, 95 \% \mathrm{CI}-4.0--1.4, \mathrm{p}<0.001$; figure 1, table 1, supplementary table S2).

$\mathrm{V}^{\prime} \mathrm{O}_{2}$ peak in $\%$ pred in patients with OSA

Mean $V^{\prime} \mathrm{O}_{2}$ peak in OSA patients expressed as \% pred was 89.9 (95\% CI 80.4-99.5) (figure 2, table 2).

\section{Subgroup analyses}

OSA severity

In patients with severe OSA (AHI>30 events $\left.\cdot h^{-1}\right)$, mean difference in $V^{\prime} \mathrm{O}_{2}$ peak compared with controls was $-2.5 \mathrm{~mL} \cdot \mathrm{kg}^{-1} \cdot \mathrm{min}^{-1}$ (95\% CI $-3.6--1.3, \mathrm{p}<0.001$; supplementary material, supplementary figure $\left.\mathrm{S} 1\right)$. In 

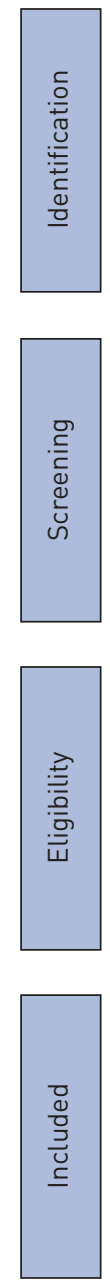

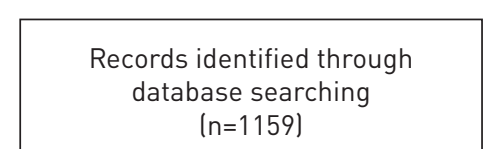
( $n=1159$ )
Additional records identified through other sources $(n=10)$

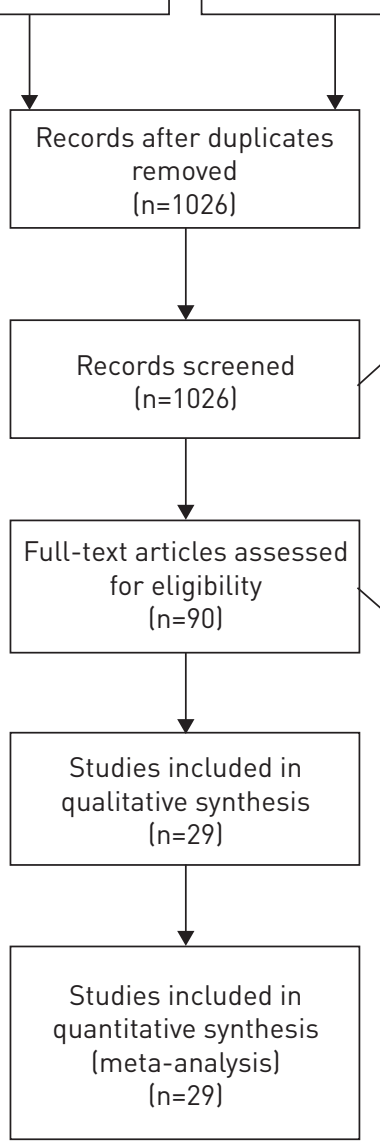

Records excluded due to eligibility criteria $(\mathrm{n}=936)$

Not OSA ( $n=475)$

OSA but not exercise tolerance

related $(n=274)$

Children ( $n=78$ )

Animal studies ( $\mathrm{n}=21$ )

Not English or French ( $n=32$ )

Reviews/editorials/letters ( $n=56$ )

Full text articles excluded with reasons $(n=61)$

No maximal exercise data $(n=23)$

No control group $(n=15)$

CPAP-treated patients $(n=3)$

OSA/CSA patients mixed $(n=7)$

OSA patients with heart failure $(n=13)$

FIGURE 3 PRISMA flow chart of articles identified and evaluated during the study selection process. OSA: obstructive sleep apnoea; CPAP: continuous positive airway pressure; CSA: central sleep apnoea.

patients with mild-moderate OSA (AHI $<30$ events.h ${ }^{-1}$ ), mean difference in $V^{\prime} \mathrm{O}_{2}$ peak compared with controls was $-1.9 \mathrm{~mL} \cdot \mathrm{kg}^{-1} \cdot \mathrm{min}^{-1}(95 \% \mathrm{CI}-3.9-0.09, \mathrm{p}=0.06)$. The difference in $V^{\prime} \mathrm{O}_{2}$ peak compared with controls did not differ significantly between patients with severe and with mild-moderate OSA $(\mathrm{p}=0.15)$.

Age

In younger OSA patients (age $<50$ years), mean difference in $V^{\prime} \mathrm{O}_{2}$ peak compared with controls was $-2.9 \mathrm{~mL} \cdot \mathrm{kg}^{-1} \cdot \mathrm{min}^{-1}(95 \% \mathrm{CI}-4.5--1.2, \mathrm{p}<0.001$; supplementary figure $\mathrm{S} 2)$. In older OSA patients (age $>50$ years), the mean difference in $V^{\prime} \mathrm{O}_{2}$ peak compared with controls was $-2.5 \mathrm{~mL} \cdot \mathrm{kg}^{-1} \cdot \mathrm{min}^{-1}$ (95\% CI $-3.7--1.2, \mathrm{p}<0.001)$. The difference in $V^{\prime} \mathrm{O}_{2}$ peak compared with controls did not differ significantly between older and younger patients $(p=0.29)$.

$B M I$

In OSA patients who were non-obese $\left(\mathrm{BMI}<30 \mathrm{~kg} \cdot \mathrm{m}^{-2}\right)$, the mean difference in $V^{\prime} \mathrm{O}_{2}$ peak compared with controls was $-4.1 \mathrm{~mL} \cdot \mathrm{kg}^{-1} \cdot \mathrm{min}^{-1}(95 \% \mathrm{CI}-5.4--2.2, \mathrm{p}<0.001$; supplementary figure $\mathrm{S} 3)$. In obese OSA patients, the mean difference in $V^{\prime} \mathrm{O}_{2}$ peak compared with obese controls without OSA was $-1.2 \mathrm{~mL} \cdot \mathrm{kg}^{-1} \cdot \mathrm{min}^{-1}(95 \% \mathrm{CI}-2.3--0.2, \mathrm{p}=0.02)$. The difference in $V^{\prime} \mathrm{O}_{2}$ peak compared with controls was significantly greater in patients who were non-obese $(\mathrm{p}<0.001)$.

Analysis of peak exercise variables

Peak heart rate was significantly lower in patients with OSA than in controls (mean difference -7.7 beat $\cdot \mathrm{min}^{-1}, 95 \%$ CI $-11.1--4.2, \mathrm{p}=0.02$; supplementary figure $\mathrm{S} 4$ ). Peak diastolic blood pressure 
TABLE 1 Summary of findings regarding exercise tolerance for studies reporting results in $\mathrm{mL} \cdot \mathrm{kg}^{-1} \cdot \mathrm{min}^{-1}$

\begin{tabular}{|c|c|c|c|c|c|c|c|c|c|c|}
\hline First author & Year & Participants & $\begin{array}{l}\text { AHI cut-off } \\
\text { events } \cdot h^{-1}\end{array}$ & $\begin{array}{l}\text { Sample } \\
\text { size } n\end{array}$ & $A H I$ events $\cdot h^{-1}$ & Age years & $\begin{array}{c}\mathrm{BMI} \\
\mathrm{kg} \cdot \mathrm{m}^{-2}\end{array}$ & Design & Sex $M / F$ & $\begin{array}{c}V^{\prime}{ }_{2 \text { peak }} \\
\mathrm{mL} \cdot \mathrm{kg}^{-1} \cdot \mathrm{min}^{-1}\end{array}$ \\
\hline \multirow[t]{2}{*}{ Alonso-Fernández [10] } & 2006 & OSA & $>10$ & 31 & $43.6 \pm 23.6$ & $53 \pm 13$ & $30.4 \pm 4$ & Baseline data of prospective study & $30 / 1$ & $25.0 \pm 6.8$ \\
\hline & & Control & $<5$ & 15 & $4 \pm 3.3$ & $48 \pm 10$ & $28.7 \pm 4.7$ & & $15 / 0$ & $25.3 \pm 7.6$ \\
\hline \multirow[t]{2}{*}{ BeitLeR [24] } & 2014 & OSA & $>15$ & 15 & $37.6(26.8-55.3)^{\#}$ & $47.9 \pm 11.5$ & $32.2 \pm 7.8$ & Cross-sectional & $12 / 3$ & $19.1 \pm 6.4$ \\
\hline & & Control & $<15$ & 19 & $1.5(0.7-5.4)^{\#}$ & $34.3 \pm 12.0$ & $28.8 \pm 6.5$ & & $10 / 9$ & $25.2 \pm 9.5$ \\
\hline \multirow[t]{2}{*}{ BUTNER [32] } & 2013 & Control & $<5$ & 43 & $2.8 \pm 1.6$ & $26 \pm 9$ & $28 \pm 6$ & Cross-sectional & $37 / 6$ & $29.4 \pm 7.7$ \\
\hline & & Moderate OSA & $5-14.9$ & 27 & $8.7 \pm 2.7$ & $30 \pm 13$ & $30 \pm 7$ & & $23 / 4$ & $26.6 \pm 6.3$ \\
\hline \multirow[t]{2}{*}{ Cavagnolli [25] } & 2014 & OSA & $>5$ & 10 & $25.7 \pm 5.4$ & $32.2 \pm 10.2$ & $27.5 \pm 1.9$ & Baseline data of prospective study & $10 / 0$ & $39.7 \pm 7.6$ \\
\hline & & Control & $<5$ & 10 & $3.5 \pm 0.5$ & $40.5 \pm 10.4$ & $26.0 \pm 3.4$ & & $10 / 0$ & $39.3 \pm 7.0$ \\
\hline \multirow{2}{*}{ Cepeda [63] } & 2015 & MetS + OSA & $\geqslant 15$ & 30 & $42 \pm 4$ & $49 \pm 1.7$ & $32 \pm 1$ & Cross-sectional & $18 / 12$ & $22.6 \pm 1.0$ \\
\hline & & MetS - OSA & $<15$ & 30 & $7 \pm 1$ & $46 \pm 1.4$ & $32 \pm 1$ & & $14 / 16$ & $23.6 \pm 1.3$ \\
\hline \multirow[t]{2}{*}{ ChIEN [27] } & 2012 & Severe OSA & $>30$ & 30 & $48.4 \pm 17.3$ & $50.5 \pm 5.7$ & $26.5 \pm 2.4$ & Baseline data of prospective study & $30 / 0$ & $25.0 \pm 3.97$ \\
\hline & & Control & & 30 & $2.7 \pm 1.3$ & $49.9 \pm 6.8$ & $25.9 \pm 2.6$ & & $30 / 0$ & $27.7 \pm 2.8$ \\
\hline \multirow[t]{2}{*}{ ChIEN [26] } & 2013 & OSA & $>30$ & 11 & $46.2 \pm 22.6$ & $50.3 \pm 5.1$ & $26.6 \pm 3.6$ & Between-group comparison & $11 / 0$ & $24.5 \pm 3.4$ \\
\hline & & Control & $<5$ & 11 & $2.7 \pm 1.1$ & $50.6 \pm 5.7$ & $26.4 \pm 3.5$ & & $11 / 0$ & $28.1 \pm 2.9$ \\
\hline \multirow{2}{*}{ HaRgens [11] } & 2008 & OSA & $>5$ & 14 & $22.7 \pm 18.5$ & $22.4 \pm 2.8$ & $32.0 \pm 3.7$ & Between-group comparison & $14 / 0$ & $27.1 \pm 4.5$ \\
\hline & & Control & $<5$ & 16 & $2.5 \pm 1.3$ & $21.4 \pm 2.6$ & $31.4 \pm 3.7$ & & $16 / 0$ & $28.0 \pm 5.8$ \\
\hline \multirow[t]{2}{*}{ INNOCENTI [64] } & 2012 & Morbidly obese + OSA & $>5$ & 8 & $51.1 \pm 24.1$ & $44 \pm 10.5$ & $44.9 \pm 7.5$ & Cross-sectional & $4 / 4$ & $17.7 \pm 7.3$ \\
\hline & & $\begin{array}{l}\text { Morbidly obese } \\
\text { control }\end{array}$ & $<5$ & 7 & $3.2 \pm 1.1$ & $34.8 \pm 10.6$ & $44.0 \pm 9.6$ & & $4 / 3$ & $19.5 \pm 5.1$ \\
\hline \multirow[t]{2}{*}{ KaLeth [12] } & 2007 & OSA & $\geqslant 5$ & 23 & $24.7 \pm 13.5$ & $45.6 \pm 10.7$ & $33.1 \pm 5.5$ & Between-group comparison & $15 / 8$ & $21.9 \pm 0.8$ \\
\hline & & Control - OSA & $<5$ & 9 & $2.5 \pm 1.6$ & $40.2 \pm 8.1$ & $29.5 \pm 5.5$ & & $2 / 7$ & $21.9 \pm 1.6$ \\
\hline \multirow[t]{2}{*}{ KLINE [65] } & 2013 & OSA & $\geqslant 15$ & 43 & $29.3 \pm 4.1$ & $46.9 \pm 1.2$ & $34.8 \pm 0.9$ & Ancillary study to RCT & $24 / 19$ & $21.6 \pm 0.8$ \\
\hline & & Control & $<5$ & 9 & $4.7 \pm 0.9$ & $46.5 \pm 2.9$ & $32.5 \pm 1.2$ & & $5 / 4$ & $22.1 \pm 2.3$ \\
\hline \multirow[t]{2}{*}{ LiN [13] } & 2006 & OSA & $\mathrm{RDI}>30$ & 20 & $44.0 \pm 8.2^{\text {ๆ }}$ & $47 \pm 7$ & $28.3 \pm 2.6$ & Between-group comparison & $18 / 2$ & $21.6 \pm 3.3$ \\
\hline & & Control & $\mathrm{RDI}<10$ & 20 & $5.1 \pm 1.6^{\pi}$ & $44 \pm 7$ & $27.6 \pm 2.7$ & & $18 / 2$ & $30.1 \pm 3.4$ \\
\hline \multirow[t]{2}{*}{ Nanas [28] } & 2010 & OSA & $\geqslant 25$ & 21 & $55 \pm 13$ & $48 \pm 11$ & $29.3 \pm 2.2$ & Between-group comparison & $21 / 0$ & $28.7 \pm 4.0$ \\
\hline & & Control & & 10 & & $46 \pm 11$ & $28.1 \pm 1.4$ & & $10 / 0$ & $34.7 \pm 6.2$ \\
\hline \multirow[t]{2}{*}{ OztuRk [30] } & 2005 & OSA & $>5$ & 19 & $46 \pm 19$ & $46.9 \pm 8.6$ & $30.7 \pm 4.6$ & Between-group comparison & $16 / 3$ & $19.8 \pm 3.1$ \\
\hline & & Control & & 11 & & $40.6 \pm 8.4$ & $28.9 \pm 3.0$ & & $7 / 4$ & $21.8 \pm 5.9$ \\
\hline \multirow[t]{2}{*}{ Rızzı [29] } & 2010 & Lean, sedentary OSA & $\geqslant 5$ & 27 & $15.4 \pm 9.2$ & $52.9 \pm 7.9$ & $23.1 \pm 1.6$ & Between-group comparison & $10 / 17$ & $28.4 \pm 10.0$ \\
\hline & & $\begin{array}{l}\text { patients } \\
\text { Controls }\end{array}$ & $<5$ & 27 & $3.1 \pm 1.1$ & $52.8 \pm 8.1$ & $22.7 \pm 1.7$ & & $10 / 17$ & $28.6 \pm 8.1$ \\
\hline \multirow[t]{4}{*}{ Rızzı [60] } & 2013 & Lean OSA & $A H I \geqslant 5$ & 22 & $22.4 \pm 11.2$ & $53.7 \pm 8.1$ & $22.1 \pm 1.6$ & Between-group comparison & $10 / 12$ & $32.1 \pm 9.5$ \\
\hline & & Lean control & $\mathrm{AHI}<5$ & 36 & $2.8 \pm 1.3$ & $50.8 \pm 6.4$ & $22.8 \pm 1.6$ & & $9 / 27$ & $30.5 \pm 7.4$ \\
\hline & & Obese OSA & $\mathrm{AHI} \geqslant 5$ & 31 & $33.3 \pm 22.9$ & $50.7 \pm 6.4$ & $33.6 \pm 2.9$ & & $7 / 24$ & $21.7 \pm 6.3$ \\
\hline & & Obese control & $A H \mathrm{I}<5$ & 26 & $2.9 \pm 1.5$ & $49.1 \pm 7.6$ & $33.4 \pm 2.6$ & & $5 / 21$ & $24.7 \pm 7.5$ \\
\hline \multirow[t]{2}{*}{ TRYFON [31] } & 2004 & OSA & $>5$ & 17 & $33.3 \pm 22.4$ & $35(22-45)^{\#}$ & $34.7 \pm 7.6$ & Between-group comparison & $17 / 0$ & $24.6 \pm 7.2$ \\
\hline & & Control & $<5$ & 10 & N/A & $35(28-54)^{\#}$ & $32.5 \pm 4.3$ & & $6 / 4$ & $23.1 \pm 5.9$ \\
\hline \multirow[t]{2}{*}{ VANHECKE [34] } & 2008 & Morbidly obese + OSA & $\mathrm{AHI}>15$ or & 42 & $32.5 \pm 26.6$ & $46.2 \pm 11.0$ & $50.5 \pm 9.4$ & Between-group comparison & $13 / 29$ & $17.6 \pm 4.2$ \\
\hline & & Morbidly obese - OSA & $\begin{array}{l}\text { AHI>5 with } \\
E S S>10\end{array}$ & 50 & $2.5 \pm 2.3$ & $45.0 \pm 8.7$ & $47.2 \pm 9.1$ & & $15 / 35$ & $21.1 \pm 3.8$ \\
\hline \multirow{2}{*}{ VANUXEm [16] } & 1997 & OSA & $>10$ & 11 & $25.6 \pm 1.2$ & $47.8 \pm 4.1$ & $26.6 \pm 1.0$ & Between-group comparison & N/A & $26.4 \pm 1.2$ \\
\hline & & Control & & 9 & & $41.9 \pm 3.1$ & $26.4 \pm 1.2$ & & & $33.2 \pm 1.4$ \\
\hline
\end{tabular}

Data are presented as $n$ or mean \pm SD, unless otherwise stated. AHI: apnoea-hypopnea index; BMI: body mass index; $M$ : male; F: female; $V^{\prime} O_{2}$ peak: peak oxygen consumption; OSA: obstructive sleep apnoea; MetS: metabolic syndrome; RDI: respiratory disturbance index; ESS: Epworth Sleepiness Scale; RCT: randomised controlled trial; N/A: data not available. \#: median (interquartile range); " : RDI provided by the investigators instead of AHI. 
TABLE 2 Summary of findings regarding exercise tolerance for studies reporting results in \% pred

\begin{tabular}{|c|c|c|c|c|c|c|c|c|c|c|}
\hline First author & Year & $\begin{array}{l}\text { AHI cut-off } \\
\text { events } \cdot h^{-1}\end{array}$ & $\begin{array}{l}\text { Sample } \\
\text { size } n\end{array}$ & $\begin{array}{c}\text { AHI } \\
\text { events } h^{-1}\end{array}$ & $\begin{array}{l}\text { Age } \\
\text { years }\end{array}$ & $\begin{array}{c}\mathrm{BMI} \\
\mathrm{kg} \cdot \mathrm{m}^{-2}\end{array}$ & Design & $\begin{array}{l}\text { Sex } \\
\text { M/F }\end{array}$ & $\begin{array}{l}\text { Equation } \\
\text { for } \% \text { pred }\end{array}$ & $\begin{array}{l}V^{\prime} 0_{2} \text { peak } \% \\
\text { pred }\end{array}$ \\
\hline ACKEL-D’ELIA [66] & 2012 & $>15$ & 12 & $40.5 \pm 22.9$ & $48.4 \pm 9.2$ & $28.0 \pm 3.1$ & Baseline data of prospective study & $13 / 0$ & $\mathrm{~N} / \mathrm{A}$ & $97.8 \pm 12.7$ \\
\hline AguilLaRd [67] & 1998 & $>5$ & 32 & $53.1 \pm 34.2$ & $47.1 \pm 10.1$ & $35.2 \pm 7.2$ & Cross-sectional & $27 / 5$ & Wasserman & $91.3 \pm 19.7$ \\
\hline Alonso-FeRnández [10] & 2006 & $>10$ & 31 & $43.6 \pm 23.6$ & $53 \pm 13$ & $30.4 \pm 4$ & Baseline data of prospective study & $30 / 1$ & ATS & $99.8 \pm 20.6$ \\
\hline BEITLER [24] & 2014 & $>15$ & 15 & $37.6(26.8-55.3)^{\#}$ & $47.9 \pm 11.5$ & $32.2 \pm 7.8$ & Cross-sectional & $12 / 3$ & Wasserman & $70.1 \pm 17.5$ \\
\hline CintRa [68] & 2009 & $>5$ & $\begin{array}{l}32 \\
30\end{array}$ & $\begin{array}{l}32.5 \pm 23.6 \\
33.9 \pm 27.6\end{array}$ & $\begin{array}{c}57.2 \pm 10.9 \\
60.5 \pm 7.4\end{array}$ & $\begin{array}{l}27.8 \pm 4.2 \\
28.4 \pm 6.3\end{array}$ & $\begin{array}{l}\text { Between-group comparison } \\
\text { (men versus women) }\end{array}$ & $\begin{array}{l}32 / 0 \\
0 / 30\end{array}$ & $\mathrm{~N} / \mathrm{A}$ & $\begin{array}{c}116.6 \pm 27.7 \\
91.3 \pm 28.7\end{array}$ \\
\hline Goel [69] & 2015 & $\begin{array}{l}>20 \\
>20\end{array}$ & $\begin{array}{c}15 \\
5\end{array}$ & $\begin{array}{c}35.3 \pm 17 \\
36.7 \pm 23.9\end{array}$ & $\begin{array}{l}56.5 \pm 9.1 \\
57.8 \pm 9.7\end{array}$ & $\begin{array}{l}30.7 \pm 5.2 \\
32.7 \pm 7.4\end{array}$ & Baseline data of prospective study & $\begin{array}{l}11 / 4 \\
5 / 0\end{array}$ & ATS & $\begin{array}{l}51.0 \pm 9.2 \\
46.6 \pm 15.1\end{array}$ \\
\hline INNOCENTI [64] & 2012 & $>5$ & 8 & $51.1 \pm 24.1$ & $44 \pm 10.5$ & $44.9 \pm 7.5$ & Cross-sectional & $4 / 4$ & Jones & $109.5 \pm 14.9$ \\
\hline MAEDER [49] & 2009 & $>5$ & 40 & $37(20-65)^{\#}$ & $50 \pm 9$ & $30.3 \pm 4.5$ & Cross-sectional & $35 / 5$ & ATS & $114 \pm 30$ \\
\hline MaEder [70] & 2007 & $>5$ & 63 & $30.3(13.0-51.7)^{\#}$ & $49.2 \pm 9.8$ & $30.1 \pm 4.8$ & Retrospective analysis & $54 / 9$ & ATS & $116 \pm 28$ \\
\hline MaEder [71] & 2008 & $>5$ & 89 & $34(17-53)^{\#}$ & $49.5 \pm 9.7$ & $30.2 \pm 4.6$ & Retrospective analysis & $78 / 11$ & ATS & $110 \pm 27$ \\
\hline NANAS [28] & 2010 & $\geqslant 25$ & 21 & $55 \pm 13$ & $48 \pm 11$ & $29.3 \pm 2.2$ & Between-group comparison & $21 / 0$ & $\mathrm{~N} / \mathrm{A}$ & $88.2 \pm 13.6$ \\
\hline OztURK [30] & 2005 & $>5$ & 19 & $46 \pm 19$ & $46.9 \pm 8.6$ & $30.7 \pm 4.6$ & Between-group comparison & $16 / 3$ & N/A & $75 \pm 10$ \\
\hline \multirow[t]{2}{*}{ OzSARAC [53] } & 2014 & $>5$ & 33 & $40.7 \pm 19.6$ & $48.8 \pm 9.2$ & $31.3 \pm 3.8$ & Baseline data of prospective study & $28 / 5$ & ATS & $87.4 \pm 21.8$ \\
\hline & & $>5$ & 32 & $35.2 \pm 26.4$ & $41.7 \pm 10.9$ & $32.2 \pm 5.8$ & & $29 / 3$ & & $78.3 \pm 18.3$ \\
\hline Pendharkar [52] & 2011 & $\geqslant 15$ & 15 & $48.1 \pm 33.1$ & $49 \pm 6$ & $42.6 \pm 8.8$ & Baseline data of prospective study & $6 / 9$ & ATS & $60^{9}$ \\
\hline PRZYBYLoWSKI [72] & 2007 & $>30$ & 111 & $47.2 \pm 23.1$ & $50.2 \pm 10.0$ & $31.0 \pm 4.6$ & Cross-sectional & $109 / 2$ & Wasserman & $85.3 \pm 17.8$ \\
\hline QUADRI [50] & 2017 & $>30$ & 12 & $45.4 \pm 14.9$ & $58.0 \pm 9.7$ & $33.3 \pm 5.2$ & Baseline data of intervention & $8 / 4$ & Wasserman & $95.3 \pm 7.6$ \\
\hline Rızzı [29] & 2010 & $\geqslant 5$ & 27 & $15.4 \pm 9.2$ & $52.9 \pm 7.9$ & $23.1 \pm 1.6$ & Between-group comparison & $10 / 17$ & $\mathrm{~N} / \mathrm{A}$ & $122 \pm 75$ \\
\hline TAPAN [73] & 2016 & $>30$ & 31 & $54.2 \pm 3.7$ & $53.4 \pm 1.5$ & N/A & Baseline data of prospective study & $27 / 4$ & N/A & $61.0 \pm 2.2$ \\
\hline
\end{tabular}

Data are presented as $\mathrm{n}$ or mean \pm SD, unless otherwise stated. $\mathrm{AHI}$ : apnoea-hypopnea index; $\mathrm{BMI}$ : body mass index: $\mathrm{M}$ : male; $\mathrm{F}$ : female; $\%$ pred: percentage predicted; $V^{\prime}{ }_{2}$ peak: peak oxygen consumption; N/A: data not available; ATS: American Thoracic Society. ${ }^{\#}$ : median (interquartile range); ${ }^{~}$ : no standard deviation available. 
tended to be greater in patients with OSA (mean difference $4.5 \mathrm{mmHg}, 95 \%$ CI $-0.1-9.2, \mathrm{p}=0.07$; supplementary figure S5). Peak minute ventilation, peak $\mathrm{O}_{2}$ pulse and peak systolic blood pressure did not differ between OSA patients and controls (supplementary figures S6, S7, S8).

\section{Quality assessment}

Studies reporting $\mathrm{V}^{\prime} \mathrm{O}_{2}$ peak in $\mathrm{mL} \cdot \mathrm{kg}^{-1} \cdot \mathrm{min}^{-1}$

The selection of patients with OSA was conducted consecutively in eight of the 19 studies [24-29]. Controls were selected from the community in five of the 19 studies [11, 13, 24, 25, 29] and the absence of OSA in controls was confirmed via polysomnography or polygraphy in all but three studies [16, 30, 31]. Only two studies out of 19 did not control for co-morbidities, age and BMI [24, 32]. In four studies, authors reported at least two criteria to define the exercise test as maximal $[16,26,27,30]$. In all the included studies, OSA patients and controls did the same maximal exercise protocol for the measurement of $V^{\prime} \mathrm{O}_{2}$ peak.

In studies of higher quality (i.e. 6 or 7 stars on the modified NOS scale), the mean difference in $V^{\prime} \mathrm{O}_{2}$ peak was $-3.6 \mathrm{~mL} \cdot \mathrm{kg}^{-1} \cdot \mathrm{min}^{-1}(95 \% \mathrm{CI}-5.7--1.5, \mathrm{p}<0.001)$ while in lower quality studies (i.e. 4 or 5 stars), the mean difference in $V^{\prime} \mathrm{O}_{2}$ peak was $-2.1 \mathrm{~mL} \cdot \mathrm{kg}^{-1} \cdot \mathrm{min}^{-1}(95 \% \mathrm{CI}-3.7--0.5, \mathrm{p}=0.01)$ between OSA patients and controls (supplementary figure S9).

\section{Sensitivity analysis}

We observed high heterogeneity $\left(\mathrm{I}^{2}=85.7 \%\right)$ across all studies reporting $V^{\prime} \mathrm{O}_{2}$ peak in $\mathrm{mL} \cdot \mathrm{kg}^{-1} \cdot \mathrm{min}^{-1}$ (supplementary figure S10). Using the leave-one-out method, there was no difference in the final estimation of the mean difference. To explain this heterogeneity, a meta-regression was performed using the following moderators as binary variables in the model: AHI $>30$, age $>50$ years, BMI $>30 \mathrm{~kg} \cdot \mathrm{m}^{-2}$ and $>80 \%$ men in the study. The results showed that the following criteria significantly contributed to the heterogeneity (estimate $\pm \mathrm{SD})$ : AHI $>30(-1.23 \pm 0.48, \mathrm{p}=0.001)$ and $\mathrm{BMI}>30(2.84 \pm 1.32, \mathrm{p}=0.03)$. The selective reporting bias was not significant $(\mathrm{p}=0.19)$. Residuals were normally distributed and the final random effects meta-analysis can be considered acceptable. The Egger test showed no selective reporting bias $(\mathrm{p}=0.50)$.

\section{Discussion}

Main results

This is the first meta-analysis to provide a comprehensive overview of the maximal exercise capacity of patients with OSA. In summary, we showed that the $V^{\prime} \mathrm{O}_{2}$ peak (expressed in $\mathrm{mL} \cdot \mathrm{kg}^{-1} \cdot \mathrm{min}^{-1}$ ) of patients with OSA was significantly lower than that of matched controls without OSA. Prior studies over the past 20 years have provided conflicting results regarding the effect of OSA on maximal exercise capacity. The limited data have been derived from small studies which were not adequately designed to discern the effects of the severity of OSA, BMI and age on maximal exercise capacity. By summarising all the available literature (19 studies for $V^{\prime} \mathrm{O}_{2}$ peak in $\mathrm{mL} \cdot \mathrm{kg}^{-1} \cdot \mathrm{min}^{-1}$ and 18 studies for $V^{\prime} \mathrm{O}_{2}$ peak in $\%$ pred), the present meta-analysis confirms the significant impact of OSA on $V^{\prime} \mathrm{O}_{2}$ peak. Patients with OSA presented with $V^{\prime} \mathrm{O}_{2}$ peak values that were $2.7 \mathrm{~mL} \cdot \mathrm{kg}^{-1} \cdot \mathrm{min}^{-1}$ lower than those of controls. In a subgroup analysis, non-obese patients $\left(\mathrm{BMI}<30 \mathrm{~kg} \cdot \mathrm{m}^{-2}\right.$ ) versus obese patients presented $V^{\prime} \mathrm{O}_{2}$ peak values that were -4.1 and $-1.2 \mathrm{~mL} \cdot \mathrm{kg}^{-1} \cdot \mathrm{min}^{-1}$, respectively, lower than controls. The reduction in maximal exercise capacity associated with OSA, especially in leaner patients, can be considered clinically significant because it has been shown that an increase in $V^{\prime} \mathrm{O}_{2}$ peak of $3.5 \mathrm{~mL} \cdot \mathrm{kg}^{-1} \cdot \mathrm{min}^{-1}$ is associated with considerable improvements in survival (10-25\%) [9]. This result suggests that the effect of OSA on $V^{\prime} \mathrm{O}_{2}$ peak is more pronounced in patients who do not present with other mechanisms that are known to decrease $V^{\prime} \mathrm{O}_{2}$ peak (i.e. obesity). Furthermore, the reduced $V^{\prime} \mathrm{O}_{2}$ peak in OSA patients was associated with lower maximal heart rate and slightly higher peak diastolic blood pressure but similar maximal minute ventilation compared to controls, suggesting some potential altered cardiovascular responses to exercise.

\section{Potential physiological mechanisms}

Several potential physiological mechanisms have been suggested to explain impaired maximal exercise capacity in OSA patients. In the present meta-analysis, heart rate at peak exercise was significantly lower in OSA patients than in controls, suggesting chronotropic incompetence. For example, KALETH et al. [12] reported that OSA patients exhibited a mean peak exercise heart rate that was $86.5 \%$ of the age-adjusted predicted maximal heart rate compared to $93.5 \%$ for controls. A number of other studies have reported this chronotropic impairment during exercise in OSA patients $[12,27,28,33,34]$ and it has been hypothesised that the impairment may be due to downregulated beta-adrenergic receptors consequent to sympathetic hyperactivity [35]. 
We did not find any differences in peak minute ventilation, peak $\mathrm{O}_{2}$ pulse or peak systolic blood pressure between OSA patients and controls. However, peak diastolic blood pressure tended to be greater in patients with OSA $(p=0.07)$, which may suggest impaired peripheral vasodilation $[36,37]$. Previous results showed that normotensive OSA patients develop elevated diastolic blood pressure at an earlier stage during exercise compared to controls [31]. This finding is important because it has been shown that diastolic hypertension during exercise is a risk factor for the subsequent development of hypertension [38] and may also constitute a limiting factor for maximal exercise capacity [39].

Other potential mechanisms that we were unable to meta-analyse owing to limited data include decreased maximal lactate concentration and delayed lactate elimination. This has been observed in OSA patients during exercise when compared to age- and BMI-matched controls and may suggest impaired glycolytic and oxidative metabolism, respectively [16]. The reduction in maximal exercise capacity in OSA patients has also been potentially attributed to abnormalities of the skeletal muscles. For example, muscle biopsy studies have demonstrated structural and bioenergetics changes in skeletal muscle fibre in OSA patients [40].

OSA patients may exhibit excessive daytime somnolence, which can affect the ability to achieve a maximal exercise workload. Several studies have indeed demonstrated reduced exercise performance under conditions of sleep deprivation $[41,42]$. A recent study from our group in healthy men showed that sleep deprivation reduced exercise time to task failure and increased the rating of perceived exertion during exercise testing [43]. Therefore, it is possible that excessive daytime sleepiness contributes to a reduced maximal exercise capacity in OSA patients.

Another potential mechanism that may contribute to the differences observed between OSA patients and controls is habitual physical activity levels. It is well known that habitual physical activity levels influence maximal exercise capacity [6]. Furthermore, a number of observational studies have reported low levels of objectively measured physical activity in OSA patients [44-46]. Only a small number of studies [10, 11, 13, 31] included in the present meta-analysis reported physical activity levels in OSA and controls. Therefore, we cannot exclude the possibility that patients with OSA had lower levels of physical activity than controls and that this contributed to their lower $V^{\prime} \mathrm{O}_{2}$ peak [47].

Controlling for BMI is imperative in studies assessing maximal aerobic capacity. All the studies included in the meta-analysis except one matched OSA patients with controls with respect to weight or BMI. However, when we compiled the BMI data across all studies, we found that the mean BMI of OSA patients was slightly but significantly greater than that of control patients $\left(+1.02 \mathrm{~kg} \cdot \mathrm{m}^{-2}, 95 \%\right.$ CI $0.49-1.54, \mathrm{p}=0.0002$; supplementary figure S11). Therefore, we cannot exclude the possibility that differences in BMI between studies may have contributed, at least in part, to the difference in maximal aerobic capacity we observed.

Differences in co-morbidities and medication intake between OSA patients and controls can also contribute to differences in exercise responses (e.g. heart rate, systolic and diastolic blood pressure) and, ultimately, $V^{\prime} \mathrm{O}_{2}$ peak. However, because the majority of studies included in the present meta-analysis had the same inclusion/exclusion criteria with respect to co-morbidities for OSA patients and controls, we do not think that co-morbidities contributed to the differences observed in $V^{\prime} \mathrm{O}_{2}$ peak between OSA patients and controls. Regarding medication, this was more scarcely reported but because co-morbidities were matched, one can expect few differences in medication intake (supplementary table S2).

Evaluating the effect of treatment of OSA on exercise responses can provide useful information concerning the effects of OSA on maximal exercise capacity. Several studies have examined this question but provide conflicting results. Although an improvement in maximal exercise performance has been observed after varying treatment durations from 1 week to 8 months [48-51], other studies have not reported enhanced $V^{\prime} \mathrm{O}_{2}$ peak after 1-3 months of continuous positive airway pressure (CPAP) treatment $[10,52,53]$. These inconsistencies may be due to differences in treatment adherence, disease severity or physical activity levels. In two of the aforementioned studies, a significant correlation was found between AHI reduction and increase in $V^{\prime} \mathrm{O}_{2}$ peak after CPAP $[48,50]$. Recently, 2 months of CPAP was shown to increase maximal exercise capacity and this was associated with decreased sympathetic hyperactivity assessed by heart rate variability. In this study, the authors ensured that the patients enrolled in the study did not change their BMI or lifestyle during the study (i.e. physical activity levels assessed via questionnaire) [50]. There is evidence that excessive sympathetic activation, as observed in chronic heart failure at baseline and during exercise, may contribute to limiting maximal exercise capacity through muscle energy metabolism and perfusion [54].

\section{Sources of heterogeneity between studies}

The high level of heterogeneity observed in the present meta-analysis can be explained by several factors including the variability of $V^{\prime} \mathrm{O}_{2}$ peak measurements, the type of study (i.e. prospective, cross-sectional, between-group comparisons) and other factors such as patient characteristics. 
Differences in protocols and criteria used to determine $V^{\prime} \mathrm{O}_{2}$ peak can be a source of variability between studies [55]. However, our methodological quality assessment scale did take into account specific elements regarding the maximal exercise testing.

The differences in $V^{\prime} \mathrm{O}_{2}$ peak \% pred values can be partly attributed to the different prediction equations used to calculate maximal aerobic capacity. Predicted $V^{\prime} \mathrm{O}_{2}$ peak equations allow a normalised evaluation of maximal exercise capacity depending on age, height and sex but some variability has been reported depending on the equation used [56]. In the present meta-analysis, the equation used varied across studies, with four studies reporting Wasserman's equation [57], eight studies citing the American Thoracic Society (ATS) guidelines [58] and one study citing Jones [59]; five did not provide information. Data in obese and non-obese adults have shown that the quantification of maximal exercise capacity in \% pred values varies depending on the equation used [58]. Hence, care has to be taken to select the most appropriate equation, especially in obese individuals, to evaluate maximal aerobic capacity in OSA patients because some prediction equations can over- or underestimate maximal exercise capacity.

The studies included in our meta-analysis did not allow us to draw conclusions regarding the effect of sex on maximal exercise capacity in OSA patients. Most studies evaluated men and women; however, four studies did not adequately sex-match OSA and control groups [24, 31, 44,60] and this may have influenced the difference in $V^{\prime} \mathrm{O}_{2}$ peak we observed between groups. To examine this, we re-analysed the data by excluding these four studies. The effect of OSA on $V^{\prime} \mathrm{O}_{2}$ peak remained similar, which suggests that differences in sex did not contribute significantly to the results observed in this meta-analysis. Another issue regarding sex differences is whether OSA affects $V^{\prime} \mathrm{O}_{2}$ peak differently in men versus women. Because no study to date has evaluated women only, our meta-analysis cannot determine whether sex has an influence on the effect of OSA on $V^{\prime} \mathrm{O}_{2}$ peak. A meta-analysis on individual data would allow a clearer picture on the effects of sex on maximal exercise capacity in OSA patients.

Another potential source of heterogeneity across studies was the physical activity level of patients. As mentioned previously, physical activity levels were only reported in a few studies and one cannot exclude the possibility that differences in physical activity levels between OSA patients and controls may explain, at least in part, the differences in $V^{\prime} \mathrm{O}_{2}$ peak we observed between groups.

The time delay between OSA diagnosis and exercise test was not available for the included studies. Moreover, the duration of exposure to OSA prior to diagnosis was unknown and is difficult to determine, although it may influence the amplitude of the effect of OSA on $V^{\prime} \mathrm{O}_{2}$ peak.

\section{Clinical implications}

The results from the present meta-analysis showing a reduction in maximal exercise capacity in OSA patients have important clinical implications. Our results shed light on the debilitating consequences of OSA on maximal exercise capacity, even in patients with OSA of mild-moderate severity. Furthermore, our results show that maximal exercise capacity is most affected in non-obese OSA patients. This highlights the importance of adapted treatment for these patients and also suggests that maximal exercise testing could be used in routine evaluation. Maximal exercise capacity testing has long been considered an effective tool to identify individuals at risk for cardiovascular disease [61]. While the impact of CPAP therapy on maximal exercise capacity remains to be elucidated with future well-designed studies, other interventions, which have been shown to improve maximal exercise capacity, should be promoted in patients with OSA. For example, exercise-based rehabilitation has been shown to not only reduce the severity of OSA and improve daytime symptoms of sleepiness, but also significantly improve maximal exercise capacity [62]. In order to limit cardiovascular and metabolic morbidity in OSA patients, this type of therapy should be encouraged.

\section{Limitations}

There are several limitations to this study. While this was the first meta-analytic study to report maximal exercise capacity in OSA patients, we did observe moderate-high heterogeneity in the included studies. Although this effect was lessened when outliers were removed, there were differences between studies of different quality (low versus moderate-high). Additional high-quality controlled studies are needed to evaluate the effect of OSA on maximal exercise capacity, with a particular focus on under-represented populations such as women.

\section{Conclusion}

Maximal exercise capacity is impaired in patients with OSA when compared with controls and when expressed relative to predictive values. Maximal exercise testing may be used to help characterise the nature of cardiopulmonary stress attendant to OSA, as well as the associated cardiometabolic dysfunction, and to evaluate the effects of treatment. 
Conflict of interest: J-C. Borel has received fees for lectures, congress invitations and a grant for a study from Philips, salaries from AGIR à dom (home care provider), fees for lectures and congress invitations from Resmed, and a patent from Nomics, outside the submitted work. J-L. Pépin reports grants from Resmed, during the conduct of the study; and grants and personal fees from Resmed, Philips, Sefam, AGIR à dom and Vitalaire; grants from Fisher and Paykel, and AstraZeneca; and personal fees from Elevie and Boehringer, outside the submitted work.

Support statement: This work was supported by Endowment fund "AGIR pour les maladies chroniques" and the French National Research Agency in the framework of the "Investissements d'avenir" programme (ANR-15-IDEX-02). Funding information for this article has been deposited with the Crossref Funder Registry.

\section{References}

1 Tamisier R, Pepin JL, Remy J, et al. 14 nights of intermittent hypoxia elevate daytime blood pressure and sympathetic activity in healthy humans. Eur Respir J 2011; 37: 119-128.

2 Lavie L. Oxidative stress inflammation and endothelial dysfunction in obstructive sleep apnea. Front Biosci (Elite Ed) 2012; 4: 1391-1403.

3 Lévy P, Kohler M, McNicholas WT, et al. Obstructive sleep apnoea syndrome. Nat Rev Dis Primers 2015; 1: 15015.

4 Stickland MK, Butcher SJ, Marciniuk DD, et al. Assessing exercise limitation using cardiopulmonary exercise testing. Pulm Med 2012; 2012: 824091.

5 Blair SN. Physical inactivity: the biggest public health problem of the 21st century. Br J Sports Med 2009; 43: 1-2.

6 Kokkinos P, Myers J. Exercise and physical activity: clinical outcomes and applications. Circulation 2010; 122: $1637-1648$.

7 Myers J, McAuley P, Lavie CJ, et al. Physical activity and cardiorespiratory fitness as major markers of cardiovascular risk: their independent and interwoven importance to health status. Prog Cardiovasc Dis 2015; 57: 306-314.

8 Kodama S, Saito K, Tanaka S, et al. Cardiorespiratory fitness as a quantitative predictor of all-cause mortality and cardiovascular events in healthy men and women: a meta-analysis. JAMA 2009; 301: 2024-2035.

9 Ross R, Blair SN, Arena R, et al. Importance of assessing cardiorespiratory fitness in clinical practice: a case for fitness as a clinical vital sign: a scientific statement from the American Heart Association. Circulation 2016; 134: e653-e699.

10 Alonso-Fernández A, García-Río F, Arias MA, et al. Obstructive sleep apnoea-hypoapnoea syndrome reversibly depresses cardiac response to exercise. Eur Heart J 2006; 27: 207-215.

11 Hargens TA, Guill SG, Zedalis D, et al. Attenuated heart rate recovery following exercise testing in overweight young men with untreated obstructive sleep apnea. Sleep 2008; 31: 104-110.

12 Kaleth AS, Chittenden TW, Hawkins BJ, et al. Unique cardiopulmonary exercise test responses in overweight middle-aged adults with obstructive sleep apnea. Sleep Med 2007; 8: 160-168.

13 Lin CC, Hsieh WY, Chou CS, et al. Cardiopulmonary exercise testing in obstructive sleep apnea syndrome. Respir Physiol Neurobiol 2006; 150: 27-34.

14 Mansukhani MP, Allison TG, Lopez-Jimenez F, et al. Functional aerobic capacity in patients with sleep-disordered breathing. Am J Cardiol 2013; 111: 1650-1654.

15 Vanhecke TE, Franklin BA, Ajluni SC, et al. Cardiorespiratory fitness and sleep-related breathing disorders. Expert Rev Cardiovasc Ther 2008; 6: 745-758.

16 Vanuxem D, Badier M, Guillot C, et al. Impairment of muscle energy metabolism in patients with sleep apnoea syndrome. Respir Med 1997; 91: 551-557.

17 Liberati A, Altman DG, Tetzlaff $\mathrm{J}$, et al. The PRISMA statement for reporting systematic reviews and meta-analyses of studies that evaluate healthcare interventions: explanation and elaboration. BMJ 2009; 339: b2700.

18 Szlachcic J, Massie BM, Kramer BL, et al. Correlates and prognostic implication of exercise capacity in chronic congestive heart failure. Am J Cardiol 1985; 55: 1037-1042.

19 Wells GA, Shea B, O'Connell RA, et al. The Newcastle-Ottawa Scale (NOS) for assessing the quality of nonrandomised studies in meta-analyses. www.ohri.ca/programs/clinical_epidemiology/oxford.asp Date last accessed: December 2017.

20 Juni $\mathrm{P}$, Witschi $\mathrm{A}$, Bloch $\mathrm{R}$, et al. The hazards of scoring the quality of clinical trials for meta-analysis. JAMA 1999; 282: 1054-1060.

21 DerSimonian R, Kacker R. Random-effects model for meta-analysis of clinical trials: an update. Contemp Clin Trials 2007; 28: 105-114.

22 Higgins JP, Thompson SG, Deeks JJ, et al. Measuring inconsistency in meta-analyses. BMJ 2003; 327: 557-560.

23 Viechtbauer W. Conducting meta-analyses in R with the metafor package. J Stat Softw 2010; 36: 1-48.

24 Beitler JR, Awad KM, Bakker JP, et al. Obstructive sleep apnea is associated with impaired exercise capacity: a cross-sectional study. J Clin Sleep Med 2014; 10: 1199-1204.

25 Cavagnolli DA, Esteves AM, Ackel-D'Elia C, et al. Aerobic exercise does not change C-reactive protein levels in non-obese patients with obstructive sleep apnoea. Eur J Sport Sci 2014; 14: Suppl.1, S142-S147.

26 Chien MY, Chang YJ, Lee P, et al. Electrophysiologic changes with incremental exercise in obstructive sleep apnea. Muscle Nerve 2013; 48: 212-218.

27 Chien MY, Lee P, Tsai YF, et al. C-reactive protein and heart rate recovery in middle-aged men with severe obstructive sleep apnea. Sleep Breath 2012; 16: 629-637.

28 Nanas S, Sakellariou D, Kapsimalakou S, et al. Heart rate recovery and oxygen kinetics after exercise in obstructive sleep apnea syndrome. Clin Cardiol 2010; 33: 46-51.

29 Rizzi CF, Cintra F, Risso T, et al. Exercise capacity and obstructive sleep apnea in lean subjects. Chest 2010; 137: 109-114.

30 Ozturk LM, Metin G, Cuhadaroglu C, et al. Cardiopulmonary responses to exercise in moderate-to-severe obstructive sleep apnea. Tuberk Toraks 2005; 53: 10-19. 
31 Tryfon S, Stanopoulos I, Dascalopoulou E, et al. Sleep apnea syndrome and diastolic blood pressure elevation during exercise. Respiration 2004; 71: 499-504.

32 Butner KL, Hargens TA, Kaleth AS, et al. Association of obstructive sleep apnea severity with exercise capacity and health-related quality of life. N Am J Med Sci 2013; 5: 362-366.

33 Grote L, Hedner J, Peter JH. The heart rate response to exercise is blunted in patients with sleep-related breathing disorder. Cardiology 2004; 102: 93-99.

34 Vanhecke TE, Franklin BA, Zalesin KC, et al. Cardiorespiratory fitness and obstructive sleep apnea syndrome in morbidly obese patients. Chest 2008; 134: 539-545.

35 Nelesen RA, Dimsdale JE, Mills PJ, et al. Altered cardiac contractility in sleep apnea. Sleep 1996; 19: 139-144.

36 Kubozono T, Koike A, Nagayama O, et al. High diastolic blood pressure during exercise is associated with hypercholesterolemia in patients with coronary artery disease. Int Heart J 2005; 46: 79-87.

37 Schultz MG, La Gerche A, Sharman JE. Blood pressure response to exercise and cardiovascular disease. Curr Hypertens Rep 2017; 19: 89.

38 Singh JP, Larson MG, Manolio TA, et al. Blood pressure response during treadmill testing as a risk factor for new-onset hypertension. The Framingham heart study. Circulation 1999; 99: 1831-1836.

39 Narkiewicz K, van de Borne PJ, Cooley RL, et al. Sympathetic activity in obese subjects with and without obstructive sleep apnea. Circulation 1998; 98: 772-776.

40 Sauleda J, Garcia-Palmer FJ, Tarraga S, et al. Skeletal muscle changes in patients with obstructive sleep apnoea syndrome. Respir Med 2003; 97: 804-810.

41 Martin BJ. Effect of sleep deprivation on tolerance of prolonged exercise. Eur J Appl Physiol Occup Physiol 1981; 47: $345-354$.

42 Martin BJ, Chen HI. Sleep loss and the sympathoadrenal response to exercise. Med Sci Sports Exerc 1984; 16: 56-59.

43 Temesi J, Arnal PJ, Davranche K, et al. Does central fatigue explain reduced cycling after complete sleep deprivation? Med Sci Sports Exerc 2013; 45: 2243-2253.

44 Kline CE, Crowley EP, Ewing GB, et al. The effect of exercise training on obstructive sleep apnea and sleep quality: a randomized controlled trial. Sleep 2011; 34: 1631-1640.

45 Mendelson M, Vivodtzev I, Tamisier R, et al. CPAP treatment supported by telemedicine does not improve blood pressure in high cardiovascular risk OSA patients: a randomized, controlled trial. Sleep 2014; 37: 1863-1870B.

46 Verwimp J, Ameye L, Bruyneel M. Correlation between sleep parameters, physical activity and quality of life in somnolent moderate to severe obstructive sleep apnea adult patients. Sleep Breath 2013; 17: 1039-1046.

47 Mendelson M, Bailly S, Marillier M, et al. Obstructive sleep apnea syndrome, objectively measured physical activity and exercise training interventions: a systematic review and meta-analysis. Front Neurol 2018; 9: 73 .

48 Lin CC, Lin CK, Wu KM, et al. Effect of treatment by nasal CPAP on cardiopulmonary exercise test in obstructive sleep apnea syndrome. Lung 2004; 182: 199-212.

49 Maeder MT, Ammann P, Münzer T, et al. Continuous positive airway pressure improves exercise capacity and heart rate recovery in obstructive sleep apnea. Int J Cardiol 2009; 132: 75-83.

50 Quadri F, Boni E, Pini L, et al. Exercise tolerance in obstructive sleep apnea-hypopnea (OSAH), before and after CPAP treatment: effects of autonomic dysfunction improvement. Respir Physiol Neurobiol 2017; 236: 51-56.

51 Taguchi O, Hida W, Okabe S, et al. Improvement of exercise performance with short-term nasal continuous positive airway pressure in patients with obstructive sleep apnea. Tohoku J Exp Med 1997; 183: 45-53.

52 Pendharkar SR, Tsai WH, Eves ND, et al. CPAP increases exercise tolerance in obese subjects with obstructive sleep apnea. Respir Med 2011; 105: 1565-1571.

53 Ozsarac I, Bayram N, Uyar M, et al. Effects of positive airway pressure therapy on exercise parameters in obstructive sleep apnea. Ann Saudi Med 2014; 34: 302-307.

54 Dexler H. Reduced exercise tolerance in chronic heart failure and its relationship to neuro-hormonal factors. Eur Heart J 1991; 12: Suppl. C, 21-28.

55 Beltz NM, Gibson AL, Janot JM, et al. Graded exercise testing protocols for the determination of VO2max: historical perspectives, progress, and future considerations. J Sports Med (Hindawi Publ Corp) 2016; 2016: 3968393.

56 Ahmadian HR, Sclafani JJ, Emmons EE, et al. Comparison of predicted exercise capacity equations and the effect of actual versus ideal body weight among subjects undergoing cardiopulmonary exercise testing. Cardiol Res Pract 2013; 2013: 940170.

57 Wasserman K, Hansen JE, Sue DY, et al. Principles of Exercise Testing and Interpretation. Philadelphia, PA, Lea \& Febiger, 1987.

58 American Thoracic Society, American College of Chest Physicians. ATS/ACCP Statement on cardiopulmonary exercise testing. Am J Respir Crit Care Med 2003; 167: 211-277.

59 Jones NL. The interpretation of stage 1 exercise test results. In: Trumbold C, ed. Clinical Exercise Testing. Philadelphia, W.B. Saunders, 1988.

60 Rizzi CF, Cintra F, Mello-Fujita L, et al. Does obstructive sleep apnea impair the cardiopulmonary response to exercise? Sleep 2013; 36: 547-553.

61 Myers J, Buchanan N, Walsh D, et al. Comparison of the ramp versus standard exercise protocols. J Am Coll Cardiol 1991; 17: 1334-1342.

62 Iftikhar IH, Kline CE, Youngstedt SD. Effects of exercise training on sleep apnea: a meta-analysis. Lung 2014; 192: $175-184$

63 Cepeda FX, Toschi-Dias E, Maki-Nunes C, et al. Obstructive sleep apnea impairs postexercise sympathovagal balance in patients with metabolic syndrome. Sleep 2015; 38: 1059-1066.

64 Innocenti BG, Gigliotti F, Scano G. Obstructive sleep apnea (OSA) does not affect ventilatory and perceptual responses to exercise in morbidly obese subjects. Respir Physiol Neurobiol 2012; 183: 193-200.

65 Kline CE, Crowley EP, Ewing GB, et al. Blunted heart rate recovery is improved following exercise training in overweight adults with obstructive sleep apnea. Int J Cardiol 2013; 167: 1610-1615.

66 Ackel-D’Elia C, da Silva AC, Silva RS, et al. Effects of exercise training associated with continuous positive airway pressure treatment in patients with obstructive sleep apnea syndrome. Sleep Breath 2012; 16: 723-735. 
67 Aguillard RN, Riedel BW, Lichstein KL, et al. Daytime functioning in obstructive sleep apnea patients: exercise tolerance, subjective fatigue, and sleepiness. Appl Psychophysiol Biofeedback 1998; 23: 207-217.

68 Cintra F, Poyares D, Rizzi CF, et al. Cardiorespiratory response to exercise in men and women with obstructive sleep apnea. Sleep Med 2009; 10: 368-373.

69 Goel AK, Talwar D, Jain SK. Evaluation of short-term use of nocturnal nasal continuous positive airway pressure for a clinical profile and exercise capacity in adult patients with obstructive sleep apnea-hypopnea syndrome. Lung India 2015; 32: 225-232.

70 Maeder MT, Münzer T, Rickli H, et al. Association between heart rate recovery and severity of obstructive sleep apnea syndrome. Sleep Med 2008; 9: 753-761.

71 Maeder MT, Ammann P, Rickli H, et al. N-terminal pro-B-type natriuretic peptide and functional capacity in patients with obstructive sleep apnea. Sleep Breath 2008; 12: 7-16.

72 Przybylowski T, Bielicki P, Kumor M, et al. Exercise capacity in patients with obstructive sleep apnea syndrome. J Physiol Pharmacol 2007; 58: Suppl. 5, 563-574.

73 Tapan ÖO, Sevinç C, İtil BO, et al. Effect of nasal continuous positive airway pressure therapy on the functional respiratory parameters and cardiopulmonary exercise test in obstructive sleep apnea syndrome. Turk Toraks Dergisi 2016; 17: 1-6. 